\title{
Iteration of Holomorphic Function Systems on the Riemann Sphere
}

\author{
Rufin EYELANGOLI OKANDZE ${ }^{1}$, Vital Delmas MABONZO ${ }^{1}$ \& Dieudonné AMPINI ${ }^{2}$ \\ ${ }^{1}$ Département des Mathématiques, Ecole Normale Supérieure, Université Marien NGOUABI, Brazzaville, Congo \\ ${ }^{2}$ Département des Mathématiques, Faculté des Sciences et Technique, Université Marien NGOUABI, Brazzaville, Congo \\ Correspondence: Rufin EYELANGOLI OKANDZE, Université Marien NGOUABI, BP: 69, Brazzaville, Congo. \\ Tel: 242-06-863-06-21.
}

Received: August 3, 2018 Accepted: August 20, 2018 Online Published: September 11, 2018

doi:10.5539/jmr.v10n5p153

URL: https://doi.org/10.5539/jmr.v10n5p153

\begin{abstract}
In this work, it is shown that a set of holomorphic functions, normal in any set, remains normal in the same set under the action of another holomorphic function. And therefore, we verify that Julia's sets of two-function composition (no matter the order of composition) coincide, up to a rescaling.
\end{abstract}

Keywords: holomorphic function, normal set, the Fatou and Julia set

\section{Introduction}

In 2005, Professor SHERETOV Vladimir Gueorguevich in (Grigorief, 2005), proposes to generalize the sets of Fatou and Julia on the case of a system of holomorphic functions on the sphere of Riemann $\bar{C}$.

By applying the classical results of the complex analysis (notably the convergence of a sequence of elements, notion of a normal family (cf. for example (Shabat, 1969) and (Goluzin, 1966)), the stability of the set of Julia or of Fatou by composition with a holomorphic function is verified.

This article is dedicated to the study of some properties of such a system. Indeed, we study the influence of a holomorphic function on a holomorphic family.

Let $f^{\text {on }}$ be the $\mathrm{n}$-th iteration of the holomorphic function $f$ (which is different to any constant) of a surface of Riemann $\mathrm{S}$ defined in itself, so we call the set of Fatou for this function, the set on which the iterations family $\left\{f^{\circ n}\right\}$ will be normal (a pre-compact family of continuous functions)(cf.(Grigorief, 2005)).

We consider the system $\left\{f_{1}, f_{2}, \cdots, f_{k}\right\}$ of holomorphic functions, which induces the following series of compositions:

$$
f_{1}, f_{2} \circ f_{1}, f_{3} \circ f_{2} \circ f_{1}, \cdots, f_{k} \circ f_{k-1} \circ \cdots \circ f_{1}, \cdots
$$

For a good understanding of this article, note the following definition:

Definition 1 System (1) is normal at the point $z_{0} \in \bar{C}$, if there exists a neighborhood $U$ of this point such that, from every infinite sequence of elements of (1), we can determine a subsequence, locally and uniformly convergent, either to a holomorphic function or to infinity.

We conclude that, system (1) is normal on a set, if it is normal in every point of this set. This set will be denoted Fatou $\left\{f_{1}, \cdots, f_{k}\right\}$ (set of Fatou for system (1)) and its complementary $\bar{C} \backslash$ Fatou $\left\{f_{1}, \cdots, f_{k}\right\}$, will be denoted Julia $\left\{f_{1}, \cdots, f_{k}\right\}$ (set of Julia for system (1)).

In this paper we will limit to the study of holomorphic systems.

The following theorem plays a vital role in this present work:

Theorem 1 Consider the holomorphic applications $f_{v}, \quad v=1, \ldots, k$, on $\bar{C}$. If the family $\left\{\left(f_{k} \circ f_{k-1} \circ \ldots \circ f_{1}\right)^{\circ n}\right\}, n \in N$, is normal to the neighborhood of $z_{0}$, so for any holomorphic function $h$, the family $\left\{h \circ\left(f_{k} \circ f_{k-1} \circ \ldots \circ f_{1}\right)^{\circ n}\right\}$ it is also normal in this same neighborhood.

Proof. Let $U$ be the neighborhood of this point $z_{0}$, such that the family $\left\{\left(f_{k} \circ f_{k-1} \circ \ldots \circ f_{1}\right)^{\circ n}\right\}$ be normal in $U$.

We prove that, $\left\{h \circ\left(f_{k} \circ f_{k-1} \circ \ldots \circ f_{1}\right)^{\circ n}\right\}$ is normal in $U$.

Let's put $g=f_{k} \circ f_{k-1} \circ \ldots \circ f_{1}$. It is clear that $\mathrm{g}$ is holomorphic.According to the hypotheses of the theorem, $g^{\circ n}$ is locally and uniformly convergent in $U$, either to a holomorphic function, or to infinity.

1st case. Supposose that, $g^{\circ n}(z)$ locally and uniformly convergent to a function $f$ in $U$. So we have: 
a) if $h$ is bounded near the point $f\left(z_{0}\right)$, therefore in a neighborhood of $z_{0}$, the compound $h \circ f(z) \neq \infty$ and continue.

Let's analyze the expression $h \circ f^{\circ n}-h \circ f$ :

$h$ being holomorphic function and bounded near the point $f\left(z_{0}\right)$, then asymptotically, one can write

$$
h(w+\Delta w)=h^{\prime}(w) \Delta w+o(|\Delta w|) .
$$

$h^{\prime}$ is also holomorphic at the point $g\left(z_{0}\right)$, then $\exists M \in R$ such that, $M=\sup \left|h^{\prime}\right|$ in the neighborhood of $f\left(z_{0}\right)$. We will have the following increase:

$$
\begin{aligned}
\left|h \circ(f)^{\circ n}-h \circ f\right| & =\left|h^{\prime}(f(z))\left(g^{\circ n}(z)-f(z)\right)+o\left(g^{\circ n}(z)-g(z)\right)\right| \\
& \leq M\left|g^{\circ n}(z)-f(z)\right|+o\left(\left|g^{\circ n}(z)-g(z)\right|\right) .
\end{aligned}
$$

The right hand side of system (2) tends to zero when $n \rightarrow \infty$, because $g^{\text {on }}$ locally and uniformly converge towards $f$;

b) Assume that $h \circ f\left(z_{0}\right)=\infty$.

Let's take $p_{n}(z)=\frac{1}{h \circ g^{\circ n}(z)} . \quad p_{n}$ is well defined because, $h \circ f\left(z_{0}\right)=\infty$ is different to zero. Plus, $p_{n} \rightarrow 0$ when $n \rightarrow \infty$.

The family of functions $p_{n}$ is holomorphic and bounded in a neighborhood of zero. So for the function $p(w)=\frac{1}{h(w)}$, there exist a real $k \in R$ such that, $k=\sup \left|p^{\prime}\right|<\infty$ in the neighborhood of $f\left(z_{0}\right)$.

We have

$$
\begin{aligned}
\left|p_{n} \circ g^{\circ n}(z)-p \circ f(z)\right| & =\left|p^{\prime}(f(z))\left(g^{\circ n}(z)-f(z)\right)\right|+o\left(\left|g^{\circ n}(z)-f(z)\right|\right) \\
& \leq K\left|g^{\circ n}(z)-f(z)\right|+o\left(\left|g^{\circ n}(z)-f(z)\right|\right) .
\end{aligned}
$$

The right hand side of system (3) tends to zero locally and uniformly in $U\left(z_{0}\right)$, when $n$ tend to $\infty$. It is for this reason that $h \circ g^{\circ n}$ locally and uniformly in $U\left(z_{0}\right)$ converge to $\infty$.

2nd case. Suppose the $g^{\circ n}$ locally and uniformly tends to $\infty$ in the neighborhood $U\left(z_{0}\right)$ of the point $z_{0}$.

Let's take $G^{\circ n}=\frac{1}{g^{\circ n}}$.

It is clear that for $n \rightarrow \infty$, the $G^{\circ n}$ locally and uniformly tends to zero. As before, we prove the local and uniform convergence of the sequence $h\left(\frac{1}{G^{\circ n}}\right)$, either to a holomorphic function or to the constant $\infty$.

The Theorem 1 is then proved.

\section{Consequence.}

Let $f_{1}$ and $f_{2}$ be two holomorphic functions, then Julia sets for the functions $f_{1} \circ f_{2}$ and $f_{2} \circ f_{1}$ are coincident.

The corresponding Pyzo program: Julia's set for the function $f_{1}(z)=z^{2}+c$

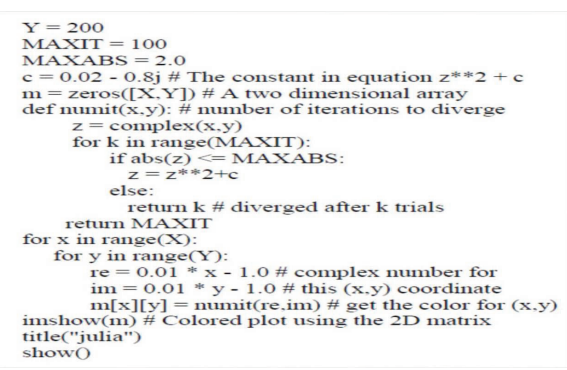

Figure 1. Pyzo program

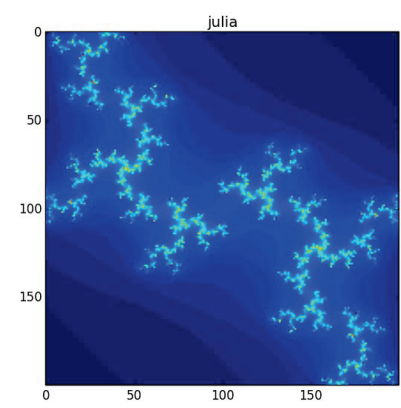

Figure 2. Julia set for the function $f_{1}(z)=z^{2}+c$ 

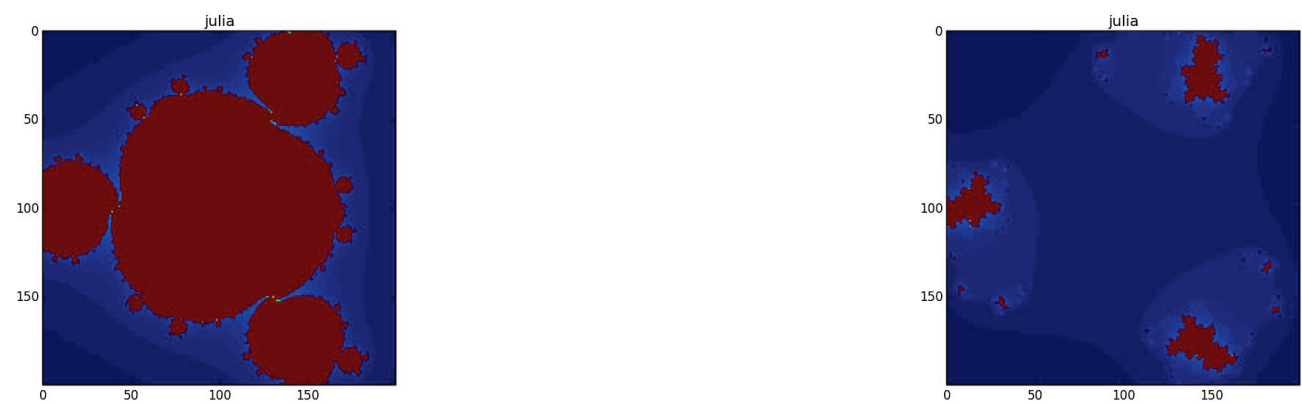

Figure 3. Julia's set for the function $f_{2}(z)=z^{3}+c$

Figure 4. Julia's set for the function $f_{1} \circ f_{2}$.

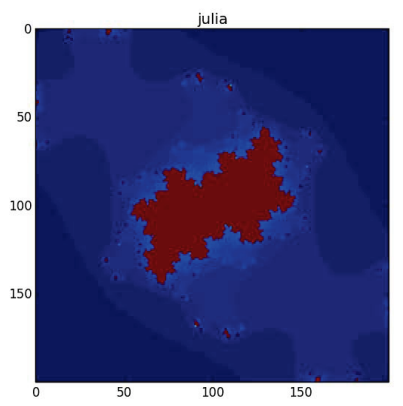

Figure 5. Julia's set for the function $f_{2} \circ f_{1}$.

Remark 1 It may be noted that Figures 4 and 5 are similar to the difference of scales. So the consequence is true.

Taking inspiration from Theorem 1, we can state the following result:

Theorem 2 The Fatou $\left\{f_{1}, \cdots, f_{k}\right\}$ set of the system (1) coincides with the Fatou set of the compound $f=f_{k} \circ \cdots \circ f_{1}$.

Proof.

1. Let us first show that Fatou $\left(f_{1}, \cdots, f_{k}\right)$ is contained in Fatou $\left(f_{k} \circ \cdots \circ f_{1}\right)=$ Fatou $(f)$.

Let $z_{0} \in \operatorname{Fatou}\left(f_{1}, \ldots, f_{k}\right)$. Then the family $f_{1}, f_{2} \circ f_{1}, \ldots$ is normal in a neighborhood $U\left(z_{0}\right)$ of point $z_{0}$. In particular, from the sequence, $f_{k} \circ \ldots \circ f_{1},\left(f_{k} \circ \ldots \circ f_{1}\right)^{\circ n}$, functions of this family, we can extract a convergent subsequence, that is to say the family of iterations $\left(f_{k} \circ \ldots \circ f_{1}\right)^{\circ n}$ is normal in the same neighborhood of $z_{0}$. Therefore Fatou $\left(f_{1}, \ldots, f_{k}\right) \subset$ Fatou $(f)$.

2. Suppose now that, $z_{0} \in \operatorname{Fatou}\left(f_{k} \circ \ldots \circ f_{1}\right)=\operatorname{Fatou}(f)$. So any family sub-sequence (1) is constituted by the elements of the form $f^{\circ n}, f_{1} \circ f^{\circ n}, f_{2} \circ f_{1} \circ f^{\circ n}, \ldots, f_{k} \circ \ldots \circ f_{1} \circ f^{\circ n}$. It is also clear that we can choose a subsequence $f_{l} \circ \ldots \circ f_{1} \circ f^{\circ n}, 1 \leq l \leq k$. Taking $h=f_{l} \circ \ldots \circ f_{1}$, the family $\left\{f^{\circ n}\right\}$ is normal, so according to Theorem $1,\left\{h \circ f^{\circ n}\right\}$ is also normal. Thus, from the elements of the family (1), it is possible to extract a subset locally and uniformly convergent, that is (1) is normal.

The theorem 2 is proved.

Remark 2 Theorems 1 and 2 could be stated relatively to Julia sets.

\section{Acknowledgements}

The authors are grateful to the referee for his/her valuable comments and suggestions which have led to an improvement of the presentation.

\section{References}

Bisi, C. (2004) On Commuting polynomial automorphisms of $\mathbb{C}^{2}$ : Publ. Mat., 48(1), 227-239.

Bisi, C. (2008) On Commuting polynomial automorphisms of $\mathbb{C}^{k}, k \geqslant 3$ : Math. Z., 258(4), 875-891. 
Blanchard, P. (1984) Complex Analytic on the Riemann Sphere : Bull. A.M.S.. 85-141.

Foster, O. (1980) Les Surfaces de Riemann.

Goluzin, G. M. (1966) Théorie gométrique des fonctions à variable complexe.

Grigorief, V. V., \& Sheretov, V. G. (2005) Certaines modifications des fractales de Mandelbrot et de Julia: Application Analyse Fonctionnelle dans la Théorie des Rapprochements. 321-2. Tver.

Milnor, J. (2000). Dynamique Holomorphe. Izhevsk.

Paitgen, H. O. (1993) La Beauté des Fractales. Images sur ordinateurs des systèmes dynamiques.

Shabat, B. V. (1969). Introduction à l'Analyse Complexe.

Sheretov, V. G. (2005) Cours sur les Surfaces de Riemann. Tver.

\section{Copyrights}

Copyright for this article is retained by the author(s), with first publication rights granted to the journal.

This is an open-access article distributed under the terms and conditions of the Creative Commons Attribution license (http://creativecommons.org/licenses/by/4.0/). 